\title{
Characteristics of multiple wavelengths L-band Brillouin-erbium comb fiber laser at low pumping powers
}

\begin{abstract}
This paper presents the characteristics of multiple wavelength L-band Brillouin-erbium comb fiber laser with intra-cavity pre-amplified Brillouin pump power technique at low pumping powers. The issue of EDF gain depletion and Brillouin gain saturation are resolved by the proposed structure. The laser structure achieves a low threshold power and is able to produce high number of output channels at low pumping powers. It produces up to 33 channels at 50 $\mathrm{mW}$ and $0.042 \mathrm{~mW}$ of $1480 \mathrm{~nm}$ pump and Brillouin pump powers, respectively.
\end{abstract}

